\title{
The effect of fragmentation and header compression on IP-based sensor networks
} (6LoWPAN)

\begin{abstract}
Internet Protocol version 6 (IPv6) over Low-power Personal Area Network (6LoWPAN) is proposed by the Internet Engineering Task Force (IETF) working group to accomplish the concept of IP-based Wireless Sensor Network (WSN). A new layer is incorporated between IPv6 network layer and 802.15.4 MAC layer, which is entitled adaptation layer. Header compression, packet fragmentation, and layer two forwarding are the main tasks of the adaptation layer. An IPv6 packet is too big regarding to the Maximum Transmission Unit (MTU) size of 802.15.4 standard thus it has to be fragmented. Breaking up a big packet to number of small fragments and attaching new headers to each fragment may affect the energy level. In this paper, we place an attention on the impact of the adaptation layer on the energy consumption of a 6LoWPAN sensor node. From our analysis, we found that the adaptation layer, in particular the fragmentation process may increase the energy consumption of a sensor node by 5 to 10 percent.
\end{abstract}

Keyword: 6LoWPAN; Wireless sensor network (WSN); Adaptation layers; Sensor nodes; Energy consumption 